\title{
Development of a Direction-Free Magnetic Field Application System
}

\author{
K. Harada ${ }^{1,2,4}$, J. Endo ${ }^{1}$, N. Osakabe ${ }^{1}$, A. Tonomura ${ }^{1,2,4}$, K. Kitazawa ${ }^{3,4}$ \\ 1) Advanced Research Laboratory, Hitachi, Ltd., Hatoyama, Saitama 350-0395, Japan \\ 2) FRS, The Institute of Physical and Chemical Research (RIKEN), Wako, Saitama 351-0198, Japan \\ 3) Department of Advanced Materials Science, University of Tokyo, Tokyo 113-0033, Japan \\ 4) SORST, Japan Science and Technology Corporation (JST), Nihonbashi, Chuo-ku, Tokyo 103-0027, Japan
}

When observing magnetic materials or superconducting vortices using Lorentz microscopy [1] and electron holography [2], it is important that the direction of the electro-optical axis and the crystalline orientation of the specimens as well as the direction of the magnetic field applied can be controlled freely. In particular, the characteristics of quantum vortices (quantized magnetic flux-lines) in high-temperature superconductors are strongly dependent on crystalline orientation. The physical phenomena of the vortices and their dynamic behaviors change drastically depending on the interactions between the external magnetic field and the crystalline orientation of the materials. The ability to change the parameters of directions during observations makes it possible to obtain much more information and increase the accuracy of experiments. For this purpose, we developed a new magnetic field system that can be applied from any direction to the specimen to be observed.

The schematic structure of the three-coil pairs used to apply the magnetic field to the specimen is shown in Fig. 1(a) and the exterior view of the manufactured direction-free magnetic field application system is shown in Fig. 1(b). The system has the following features:

(1) An external magnetic field can be applied to the specimen from any direction,

(2) The magnetic application system is independent of the sample holder,

(3) A system of compensation for electron beam deflection by the externally applied magnetic field is incorporated,

(4) The magnetic field application system and the compensation system are controllable by computer,

(5) Non-core coils are used to make a hysteresis-free magnetic field to enable more accurate control of a weak magnetic field,

(6) Superconducting feed-throughs (electric wires) are used to achieve down-sizing,

(7) The superconducting coil system has the advantages of cooling the specimen and maintaining an ultra-high vacuum around the specimen.

The magnitude of the magnetic fields applied at an electric current of $500 \mathrm{~mA}$ was $9.4 \mathrm{mT}$ horizontally and 54 mT vertically. We confirmed that the maximum magnetic fields described above could be practically applied to a specimen. In addition, the deflection of the electron beam was compensated for and adjusted to the electro-optical system below the magnetic field application system.

This system has already been installed in 1-MV [3] and 300-kV field emission electron microscopes and is now being used to observe the superconducting vortices [4,5]. Examples of observations are shown in Fig. 2. 


\section{References}

[1] K. Harada et al., Nature 360 (1992) 51.

[2] J. E. Bonevich et al., Phys. Rev. Lett. 70 (1993) 2952.

[3] T. Kawasaki et al., Appl. Phys. Lett. 76 (2000) 1342.

[4] A. Tonomura et al., Nature 412 (2001) 620.

[5] T. Matsuda et al., Science 294 (2001) 2136.

[6] The authors thank T. Katsuta, I. Matsui, S. Hayashi, T. Onai, M. Numata, T. Furutsu, S. Saitou, S. Matsunami, N. Moriya, S. Kubota, T. Matsuda and H. Kasai for their technical support.
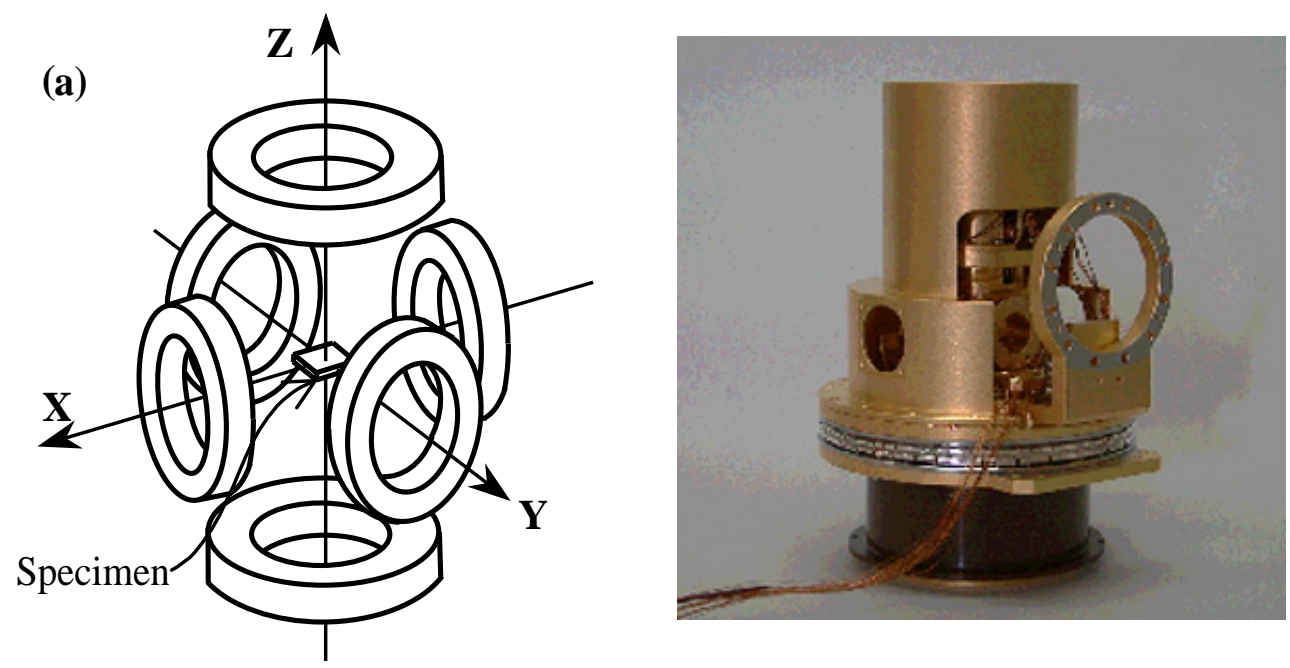

Figure 1 (a) Schematic structure of the three pairs of coils, (b) Exterior view of the manufactured system.
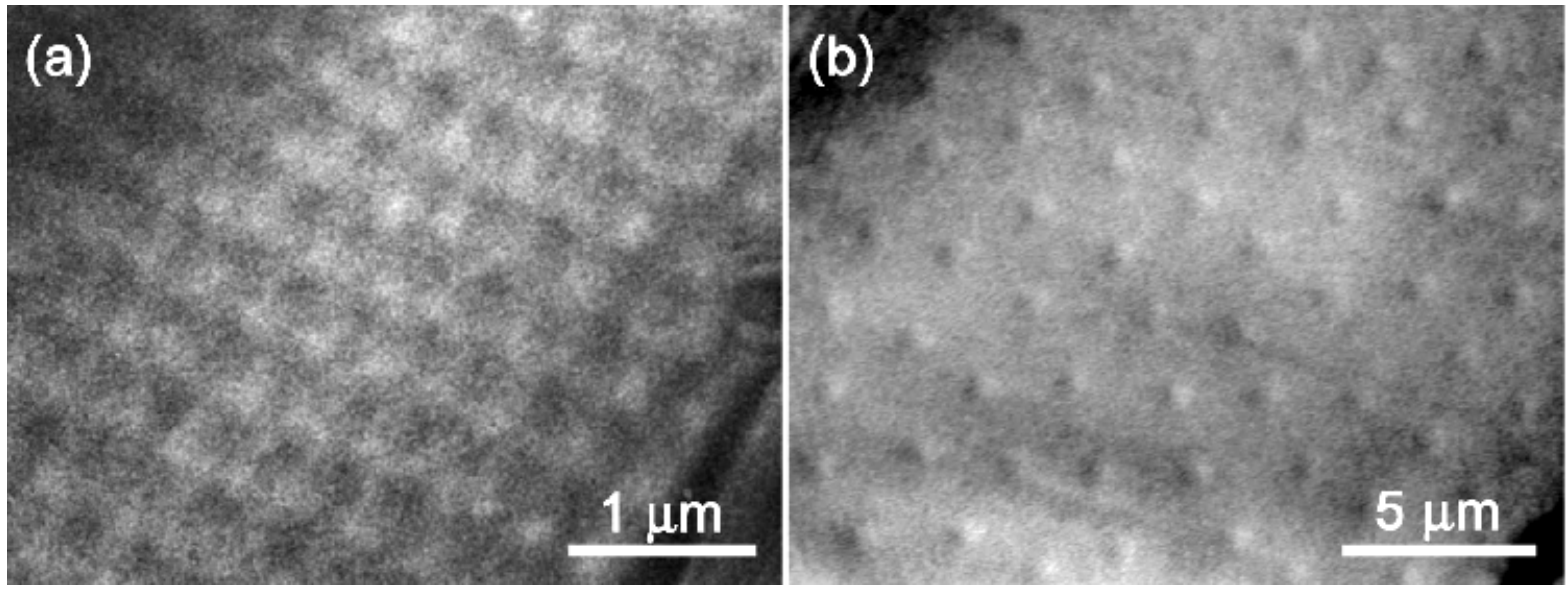

Figure 2 Lorentz micrographs of the superconducting vortices using $300-\mathrm{kV}$ field emission electron microscope; (a) Niobium (Nb) at $8 \mathrm{mT}$, (b) $\mathrm{Bi}_{2} \mathrm{Sr}_{2} \mathrm{CaCu}_{2} \mathrm{O}_{8+\delta}(\mathrm{Bi}-2212)$ at $1.4 \mathrm{mT}$. 